\title{
A novel antiplatelet antibody therapy that induces cAMP-dependent endocytosis of the GPVI/Fc receptor $\gamma$-chain complex
}

\author{
Hiroshi Takayama, ${ }^{1,2}$ Yoshitaka Hosaka, ${ }^{3}$ Kazuyuki Nakayama, ${ }^{3}$ Kamon Shirakawa, ${ }^{3}$ \\ Katsuki Naitoh, ${ }^{3}$ Tomokazu Matsusue, ${ }^{3}$ Mikihiko Shinozaki, ${ }^{3}$ Motoyasu Honda, ${ }^{3}$ Yukiko Yatagai, ${ }^{3}$ \\ Tetsushi Kawahara, ${ }^{3}$ Jiro Hirose, ${ }^{3}$ Tooru Yokoyama, ${ }^{3}$ Michiru Kurihara, ${ }^{1}$ and Shoji Furusako ${ }^{3}$ \\ 'Department of Health and Nutrition, School of Human Cultures, The University of Shiga Prefecture, Shiga, Japan. ${ }^{2}$ Department of Hematology and Oncology, \\ Graduate School of Medicine, Kyoto University, Kyoto, Japan. ${ }^{3}$ Pharmaceutical Research Center, Mochida Pharmaceutical Co. Ltd., Shizuoka, Japan.
}

\begin{abstract}
Platelet adhesion to vascular subendothelium, mediated in part by interactions between collagen and glycoprotein VI (GPVI) complexed with Fc receptor $\gamma$-chain, is crucial for thrombus formation. Antiplatelet therapy benefits patients with various thrombotic and ischemic diseases, but the safety and efficacy of existing treatments are limited. Recent data suggest GPVI as a promising target for a novel antiplatelet therapy, for example, GPVI-specific Abs that deplete GPVI from the surface of platelets. Here, we characterized GPVI-specific autoAbs (YA-Abs) from the first reported patient with ongoing platelet GPVI deficiency caused by the YA-Abs. To obtain experimentally useful human GPVI-specific mAbs with characteristics similar to YA-Abs, we generated human GPVI-specific mouse $\mathrm{mAbs}$ and selected 2 representative $\mathrm{mAbs}, \mathrm{mF1} 201$ and $\mathrm{mF1232}$, whose binding to GPVI was inhibited by YA-Abs. In vitro, $\mathrm{mF1201}$, but not $\mathrm{mF1232}$, induced human platelet activation and GPVI shedding, and mF1232 inhibited collagen-induced human platelet aggregation. Administration of mF1201 and mF1232 to monkeys caused GPVI immunodepletion with and without both significant thrombocytopenia and GPVI shedding, respectively. When a human/mouse chimeric form of mF1232 (cF1232) was labeled with a fluorescent endocytosis probe and administered to monkeys, fluorescence increased in circulating platelets and surface GPVI was lost. Loss of platelet surface GPVI mediated by cF1232 was successfully reproduced in vitro in the presence of a cAMP-elevating agent. Thus, we have characterized cAMP-dependent endocytosis of GPVI mediated by a human GPVI-specific $\mathrm{mAb}$ as what we believe to be a novel antiplatelet therapy.
\end{abstract}

\section{Introduction}

Antiplatelet therapy provides significant clinical benefits in patients with a variety of thrombotic or ischemic diseases such as myocardial infarction, unstable angina, and stroke (1). However, the safety and efficacy of existing treatments are limited, and there remains a clinical need for novel antiplatelet interventions to reduce pathological thrombus formation without promoting bleeding (2).

Over the last decade, considerable progress has been made in identifying and describing the molecular events regulating the initiation of thrombus formation. At sites of vascular damage, subendothelial matrix proteins, primarily collagen, are exposed, promoting platelet adhesion and activation through interactions with specific cell-surface receptors, leading to thrombus formation.

Several cellular collagen receptors mediate this response, and, among these, glycoprotein VI (GPVI) plays a critical role in the platelet response to collagen. GPVI is a member of the Ig superfamily (3-5), and it is constitutively associated and expressed with the $\mathrm{Fc}$ receptor $\gamma$-chain $(\mathrm{FcR} \gamma)$, an immunoreceptor tyrosine-based activating motif-bearing (ITAM-bearing) receptor (6). Upon GPVI engagement, phosphorylation of the ITAM motif of FcR $\gamma$ initiates a signaling cascade, leading to platelet activation (6-10). The

Nonstandard abbreviations used: ADP, adenosine diphosphate; cGPVI, cynomolgus GPVI; CRP, collagen-related peptide; D1, domain 1; hGPVI-Fc, hGPVI fused to human Fc; FcR $\gamma$, FcR $\gamma$-chain; GPVI, glycoprotein VI; hGPVI, human GPVI; mGPVI, mouse GPVI; PRP, platelet-rich plasma; TRAP, thrombin receptor-agonist peptide; YA-Abs, YA's auto-Abs.

Conflict of interest: The authors have declared that no conflict of interest exists. Citation for this article: J. Clin. Invest. 118:1785-1795 (2008). doi:10.1172/JCI32513. extracellular domain of GPVI contains 2 Ig-like loops of the C2 type (3-5). The extracellular domain 1 (D1) N-terminal loop is of the IgC2-1 type, and the extracellular D2 loop is of the IgC2-2 type (11). Deficiency or blockade of GPVI slightly prolongs bleeding time in both humans and mice (12-14), and this produces antithrombotic effects in several different experimental conditions (14-17). These data suggest that GPVI is a promising target for the development of novel antiplatelet therapies, but such therapies have yet to be successfully developed and/or clinically deployed.

Platelet GPVI deficiency and unresponsiveness to collagen have been first reported in a patient (YA) who developed anti-GPVI Abs in the plasma (12). Monovalent Fab fragments prepared from YA's serum IgG blocked collagen-induced platelet aggregation, but divalent $\mathrm{F}\left(\mathrm{ab}^{\prime}\right)_{2}$ fragments caused GPVI cross-linking and platelet activation. This report also suggested the possibility of specific $\mathrm{Ab}$ induced GPVI immunodepletion. This assumption was confirmed by later studies in mice $(14,18)$, and an additional patient with Abinduced GPVI deficiency was identified (19). Short-term antiplatelet effects may be safely generated by the infusion of anti-GPVI Fab fragments to block the GPVI/collagen interaction. The $\mathrm{F}\left(\mathrm{ab}^{\prime}\right)_{2}$ fragments and intact $\mathrm{Ab}$ have a longer half-life in the serum than the Fab fragments, but their divalent nature would likely activate platelets by cross-linking GPVI. Alternatively, specific Ab-induced GPVI immunodepletion could be clinically useful. However, metalloproteinase-dependent proteolytic shedding of GPVI has been demonstrated in vitro to cause considerable loss of GPVI following activation of platelets exposed to GPVI-specific Abs or ligands (20, 21). On the other hand, GPVI internalization and endocytosis may 

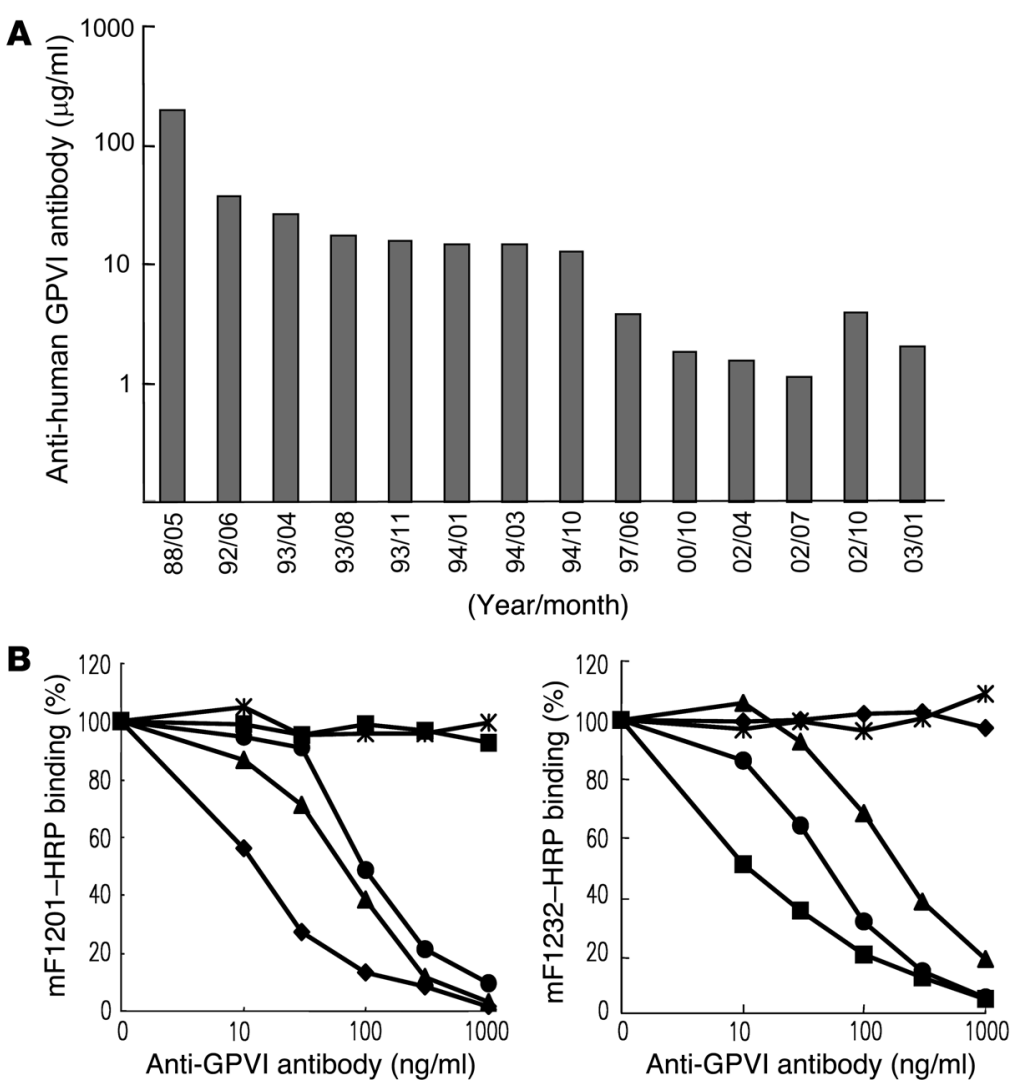

\section{Figure 1}

Characterization of YA-Abs over 15 years. (A) YA's serially diluted plasma stored over 15 years or control solutions containing known amounts of a human/mouse chimera anti-GPVI mAb were incubated with biotin-labeled hGPVIFc on streptavidin-coated plates. After washing, the bound Abs were detected using peroxidase-labeled anti-kappa and -lambda chain Abs and subsequent colorimetric reaction. The content of anti-GPVI auto-Abs in the plasma was expressed as an equivalent content of a human/ mouse chimera anti-GPVI mAb bound to hGPVI-Fc. Each bar represents the mean of duplicate determinations. (B) Both YA-Abs-88 and -03 were purified from the patient's plasma stored in 1988 and 2003, respectively, and quantified as described in Methods. Serially diluted YA-Abs-88 (triangles), YA-Abs-03 (circles), mF1201 (diamonds), mF1232 (squares), or mouse IgG (asterisks) as the competing Ab was applied to hGPVI-Fc-immobilized plates followed by the incubation with the peroxidaselabeled mF1201 (left panel) or mF1232 (right panel). After washing, the bound labeled Abs were detected by colorimetric reaction. Data are expressed as the percent of total binding of each peroxidase-labeled $\mathrm{mAb}$ without the competitor and are the mean of triplicate determinations. not be denied as another mechanism for Ab-induced GPVI immunodepletion. However, no data exist about a GPVI-specific Ab that does induce GPVI internalization and endocytosis but not GPVI shedding. Most importantly, specific Ab-induced GPVI internalization and endocytosis remains to be completely demonstrated.

With these backgrounds in mind, we aimed to obtain clinically useful mAbs against human GPVI (hGPVI) with characteristics similar to YA's auto-Abs (YA-Abs) and investigated the mechanisms underlying GPVI immunodepletion. In addition to our first presentation of nonactivating hGPVI-specific mAb-induced GPVI deficiency without significant thrombocytopenia (22), the present work reports the establishment of clinically applicable induction of the Ig-D2-specific mAb-mediated GPVI deficiency and also what we believe to be the first clarification of the mechanisms underlying such induction.

\section{Results}

Our initial approach was based upon 2 assumptions: (a) the plasma of patient YA contains auto-Abs that downregulate GPVI expression, and (b) mouse mAbs that bind the same epitope(s) on hGPVI will function similarly to YA-Abs. We first analyzed frozen plasma isolated from patient YA for the presence of anti-GPVI autoAbs using recombinant hGPVI fused to human Fc (hGPVI-Fc) as a target. As seen in Figure 1A, the concentration of anti-GPVI Abs in the plasma of YA was highest in 1988 and decreased more than 100 -fold over the course of 15 years, plateauing at 1 to $5 \mu \mathrm{g} / \mathrm{ml}$ after 2002. However, during this time, the patient's platelets remained unresponsive to collagen stimulation due to complete GPVI deficiency (23), and the number of platelets was maintained at normal levels (data not shown).
In order to characterize YA-Abs more in detail, we purified YAAbs from the patient's plasma stored in 1988 (YA-Abs-88) and in 2003 (YA-Abs-03) using sequential affinity chromatography on protein A-column and recombinant hGPVI-Fc-column and examined cross-reactivities of those YA-Abs with mouse anti-GPVI $\mathrm{mAbs}$ for binding to hGPVI-Fc as described in Methods. YA-Abs efficiently competed with several $\mathrm{mAbs}$ for binding to hGPVI-Fc, and we selected 2 representative $\mathrm{mAbs}, \mathrm{mF} 1201$ and $\mathrm{mF} 1232$, for further study. These $2 \mathrm{mAbs}$ did not cross-inhibit one another, indicating that they bind different epitopes of hGPVI. As shown in Figure 1B, mF1201 binding to hGPVI-Fc was inhibited by both YA-Abs- 88 and YA-Abs- 03 ( $\mathrm{IC}_{50}, 58 \mathrm{ng} / \mathrm{ml}$ versus $\left.89 \mathrm{ng} / \mathrm{ml}\right)$. In contrast, mF1232 binding to hGPVI-Fc was inhibited by YA-Abs-03 more strongly than YA-Abs-88 ( $\mathrm{IC}_{50}, 45 \mathrm{ng} / \mathrm{ml}$ versus $\left.136 \mathrm{ng} / \mathrm{ml}\right)$. These data suggest that YA-Abs-03 likely shares more epitopes with mF1232 than mF1201, despite the relatively low titer.

We next examined the ability of the isolated mAbs to modulate platelet function either through cross-linking-induced activation or by inhibiting collagen-induced platelet aggregation. Treatment of platelets with up to $10 \mu \mathrm{g} / \mathrm{ml}$ of $\mathrm{mF} 1201$ (in excess of reported plasma concentrations of YA-Abs isolated after 1997) induced both platelet aggregation as well as expression of the activation marker $\mathrm{CD} 62 \mathrm{P}$ in a dose-dependent manner. In contrast to this finding, mF1232 neither induced aggregation nor significant CD62P expression but dose dependently inhibited collagen-induced platelet aggregation (Figure 2, A-C). However, $10 \mu \mathrm{g} / \mathrm{ml} \mathrm{mF1232} \mathrm{did} \mathrm{not}$ inhibit platelet aggregation in response to collagen-related peptide (CRP), a synthetic peptide that acts as a GPVI-specific agonist (24), adenosine diphosphate (ADP), ristocetin and $\mathrm{vWF}$, or thrombin receptor-agonist peptide (TRAP) (Figure 2C and data not shown). 
A
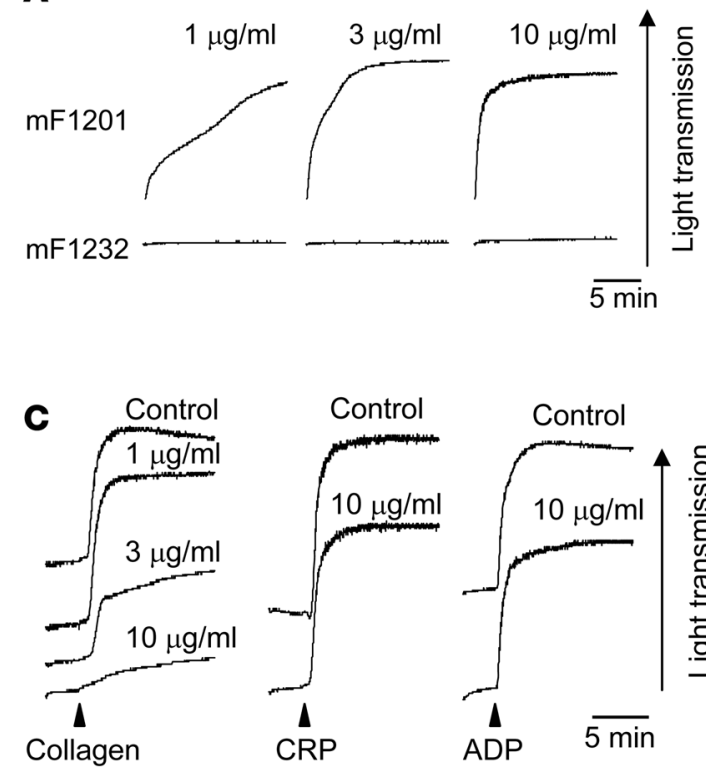

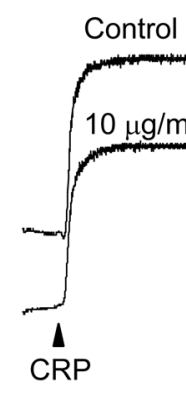

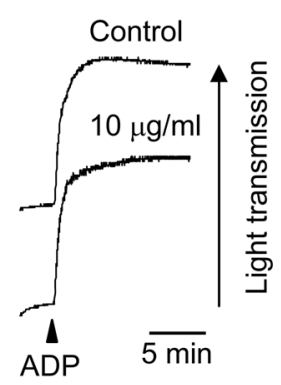
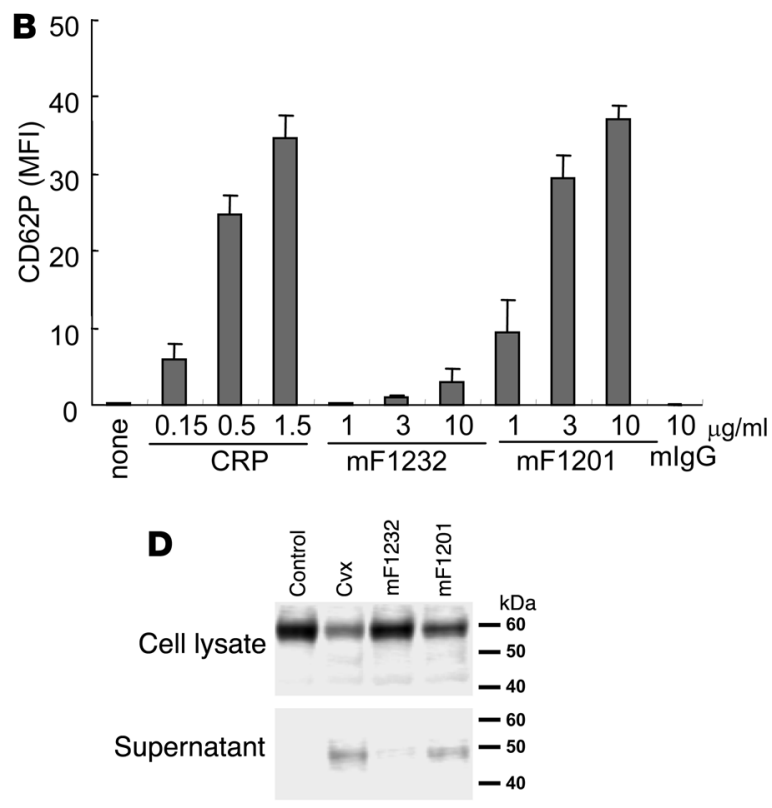

\section{Figure 2}

$\mathrm{mF1201}$, but not $\mathrm{mF1232}$, induces both platelet aggregation and shedding of surface GPVI, while mF1232 inhibits collagen-induced, but not CRP- or ADP-induced, platelet aggregation. (A) Platelet aggregation was monitored after the addition of the indicated concentrations of $\mathrm{mF} 1201$ or mF1232 into human PRP. (B) After human PRP was incubated without (none) or with the indicated concentrations of CRP, $m F 1232, \mathrm{mF1201}$, or $\mathrm{mlgG}$ for 30 minutes at $37^{\circ} \mathrm{C}$, the platelets were fixed and stained with PE-labeled anti-human CD62P Ab for flow cytometry analysis. Data represent mean \pm SEM of triplicate determinations. MFI, mean fluorescence intensity. (C) Human PRP was preincubated with the indicated concentrations of $\mathrm{mF} 1232$ for 5 minutes at $37^{\circ} \mathrm{C}$ and stimulated with $1 \mu \mathrm{g} / \mathrm{ml}$ collagen, $4 \mu \mathrm{g} / \mathrm{ml} \mathrm{CRP}$, or $5 \mu \mathrm{M}$ ADP as indicated by arrows. Traces are representatives of 2 or 3 separate experiments from different donors. (D) Washed human platelets were treated without (control) or with $0.15 \mu \mathrm{g} / \mathrm{ml}$ convulxin $(\mathrm{Cvx}), 10 \mu \mathrm{g} / \mathrm{ml} \mathrm{mF1232}$, or $10 \mu \mathrm{g} / \mathrm{ml} \mathrm{mF1201}$ for 60 minutes at $37^{\circ} \mathrm{C}$, and centrifuged to isolate platelet pellets from supernatants. Platelet pellets (cell lysate) and supernatants were analyzed by western blotting using rabbit anti-GPVI polyclonal Abs. Data are representatives of 3 separate experiments from different donors.

Metalloproteinase-dependent proteolytic shedding is thought to mediate the loss of GPVI expression from platelets exposed to GPVI-specific mAb or ligands $(20,21)$. Therefore, we examined whether either mF1201 or mF1232 induced GPVI-shedding from the platelet surface. Incubation of washed human platelets with 10 $\mu \mathrm{g} / \mathrm{ml} \mathrm{mF1201} \mathrm{resulted} \mathrm{in} \mathrm{the} \mathrm{loss} \mathrm{of} \mathrm{surface} \mathrm{GPVI,} \mathrm{accompanied} \mathrm{by}$ the appearance of its soluble cleavage product, but similar changes were not seen in platelets exposed to $\mathrm{mF} 1232$ (Figure 2D).

YA-Abs have been used to probe only hGPVI $(6-8,10,12,13,17$, $23,25)$, and we tested both $\mathrm{mF} 1201$ and $\mathrm{mF} 1232$ for their ability to react with GPVI-Fc obtained from different species. We cloned cynomolgus GPVI (cGPVI), and based on amino acid sequence alignment, it is $90 \%$ identical to hGPVI. In contrast, mouse GPVI (mGPVI) is only 63\% identical to hGPVI over the same region (Figure $3 \mathrm{~A})$. Using a competition assay, in which a signal is only obtained if there is no cross reactivity, binding of mF1201 or mF1232 to hGPVIFc was not inhibited by mGPVI-Fc (Figure 3, B and C). In contrast, cGPVI-Fc efficiently competed with hGPVI-Fc for binding to either $\mathrm{mAb}$. These data indicate that both generated anti-GPVI mAbs did not recognize mGPVI but cGPVI was bound. To further characterize the epitopes recognized by $\mathrm{mF} 1201$ and $\mathrm{mF} 1232$, we mutated different regions of hGPVI-Fc to contain the corresponding sequence as indicated in Figure 3A. Using the same competition assay, mutation of loop 9 of the Ig-D2 and mutation of either loop 4 or 5 of the Ig-D1 were found to lose their inhibitory effects on $\mathrm{mF} 1232$ and mF1201 binding to hGPVI-Fc, respectively (Figure 3, B and C).
These results were confirmed by a direct binding assay using WT and mutant hGPVI-Fc coated on a plate (Figure 3, D and E). Previous studies identified 2 distinct collagen-binding sites on hGPVI. One binding site includes Lys59 and is recognized by the 10B12 single-chain phage $\mathrm{Ab}$ (26). The other collagen-binding site is located in Ig-D1 and includes residues Gly30, Val34, and Leu36, and this site is bound by the $9012 \mathrm{mAb}$ (27). In our study, either mF1201 or mF1232 binding to hGPVI-Fc was inhibited by the mutant at loop 3, including Gly30, Val34, and Leu36 (Figure 3, B and C). However, since Lys59 is included within loop 5, we also generated a hGPVI-Fc point mutant containing the corresponding mouse residue (K59E) at this location. There was no binding of $\mathrm{mF} 1201$ to K59E mutant hGPVI-Fc (data not shown), suggesting that mF1201 recognizes the same collagen-binding site as 10B12 (26).

We next wished to investigate the ability of both $\mathrm{mAbs}$ to immunodeplete GPVI in vivo using cynomolgus monkeys as a model. We first confirmed that both mAbs affected cynomolgus platelets in a similar manner to human platelets (data not shown). When either $\mathrm{mAb}$ was i.v. injected into monkeys at a dose of $0.3 \mathrm{mg} / \mathrm{kg}$, an amount estimated to be sufficient to saturate the total surface GPVI according to previously reported GPVI expression data (28), expression of both GPVI and FcR $\gamma$ was decreased within 1 to 2 days, and this was accompanied by platelet unresponsiveness to collagen but not to ADP, ristocetin and vWF, and TRAP (Figure 4, A-C, and data not shown). Additionally, significant thrombocytopenia occurred following injection of $\mathrm{mF1201}$ but not mF1232 (Figure 4D). 
A Human Mouse Cynomolgus
L1 QSGPLPKPSL QATPSSLVPL EKPVTLRCQG

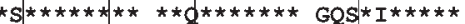
$\mathrm{R}^{\star \star \star \star \star \star \star \star \star \star \star \star \star ~} \mathrm{I}$
L3 PPGVDIYRLE KI SSSRYQDQ AVLFIPAMRR

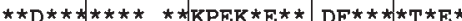

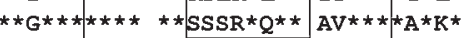
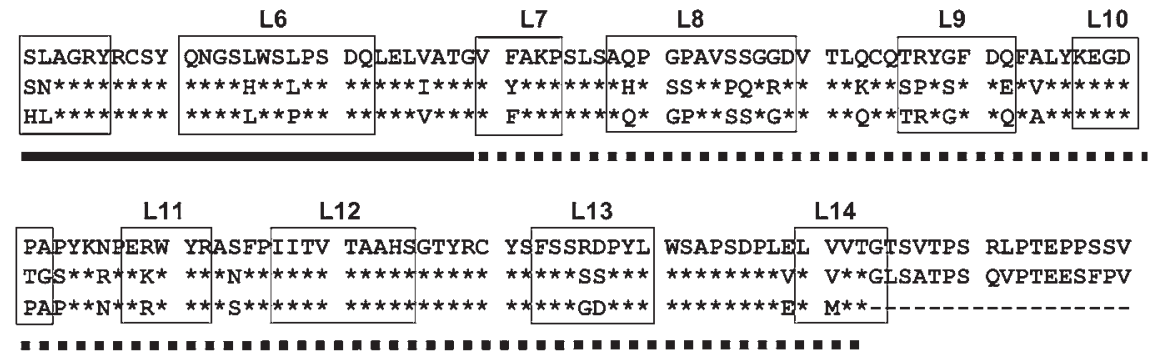

AEFSEATAEI TVSFTNKVET TETSRSITTS PKESDSPAGP ARQYYTKGN

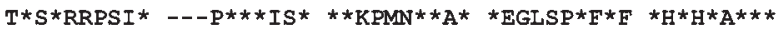

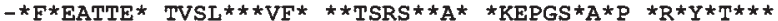
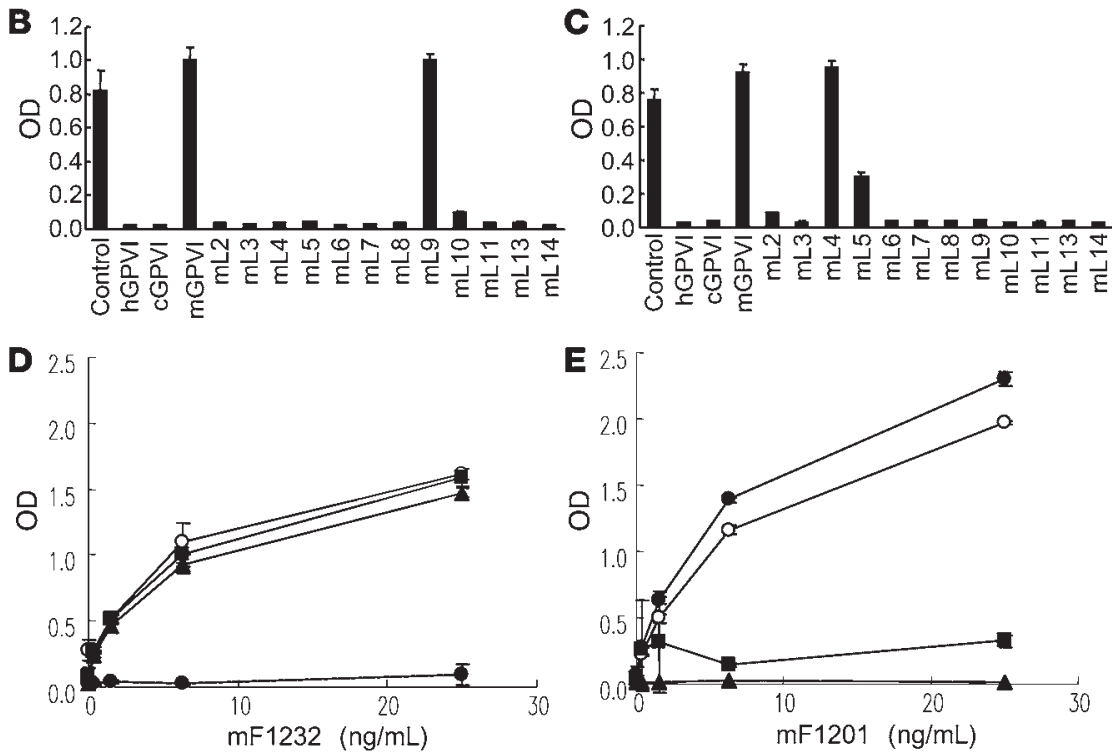

Figure 3

Binding of mF1232 and mF1201 to WT GPVI and to hGPVI mutants. (A) Amino acid alignment of the extracellular domains of GPVI from 3 species are shown. Conserved amino acids among the 3 species are indicated by stars. The Ig-D1 and -D2 regions of GPVI are underlined with solid lines and dotted lines, respectively. To construct of the extracellular portion of GPVI, a comparative molecular modeling was carried out with "Homology" (Accelrys Software Inc.) using PDB 1UGN_A and PDB 1P6F_A as a template. The regions boxed with solid lines were defined as the loop structures. The abbreviation over the box denotes each loop number. (B and C) Fifty $\mathrm{ng} / \mathrm{ml}$ of peroxidase-labeled $\mathrm{mF} 1232$ (B) or $\mathrm{mF1201}$ (C) was incubated in an hGPVI-Fc-coated immunoplate for 60 minutes at $37^{\circ} \mathrm{C}$ with $1 \mu \mathrm{g} / \mathrm{ml}$ each of the absorption antigens hGPVI, mGPVI, cGPVI, or human/mouse chimera GPVI mutants. Human/mouse chimera GPVI mutant protein is represented as "mL plus number" meaning that the amino acid sequence of loop number on hGPVI is substituted for the counter loop region of mGPVI. (D and E) Peroxidase-labeled mF1232 (D) or mF1201 (E) were incubated with hGPVI-Fc- (open circles), mL9- (filled circles), $\mathrm{mL} 4$ - (triangles), or mL5-coated (squares) plates. After incubation, the bound peroxidase-labeled $\mathrm{mAb}$ was measured. Data are presented as mean \pm SD of triplicate determinations.

Taken together, our data suggest that $\mathrm{mF} 1232$ is a more promising therapeutic agent than $\mathrm{mF} 1201$, an activating $\mathrm{mAb}$, and we constructed a human/mouse chimera of mF1232 (cF1232) for further study. When we compared $\mathrm{cF} 1232$ with $\mathrm{mF} 1232$ in assays described in Figures 2, 3, and 4, there were no differences between

these 2 reagents (data not shown). Importantly, cF1232 did not induce substantial activation of human platelets such as aggregation and CD62P expression, even at $3 \mathrm{mg} / \mathrm{ml}$, the dose maximally tested here (data not shown).

In order to monitor the behavior of cF1232 after injection into monkeys and binding to platelet surface GPVI, we used CypHer5E, a fluorescent endocytosis probe. This dye is nonfluorescent at neutral $\mathrm{pH}$, but its fluorescence increases exponentially as it passes through the acidic environment of endosomes and lysosomes, with maximum fluorescence at $\mathrm{pH} 5$, the luminal lysosomal pH $(29,30)$. After CypHer5E-labeled cF1232 was i.v. injected into monkeys, we monitored the fluorescence of CypHer5E in circulating platelets and surface GPVI expression by flow cytometry as well as the expression of GPVI/FcR $\gamma$ in whole cell lysates by immunoblotting. As shown in Figure 5A, CypHer5E fluorescence in circulating platelets rapidly increased within 1 hour after injection, and this was maintained at a maximum level thereafter. Both surface GPVI (Figure 5B) and total cellular GPVI/FcR $\gamma$ complex expression (Figure 5C) began to concomitantly decrease, and surface GPVI was reduced by approximately $80 \%$ at 6 hours after injection. We wished to confirm that $\mathrm{Ab}$ binding and uptake was dependent upon the surface expression of GPVI, and we injected monkeys with unlabeled cF1232, at a dose of $1 \mathrm{mg} / \mathrm{kg}$, to immunodeplete surface GPVI. Five days after this initial injection, monkeys were then treated with CypHer5E-labeled cF1232, and very low levels of fluorescence were seen at 3 hours after injection. This was much lower than the fluorescence seen in nonimmunodepleted monkeys (Figure 5, A and B).

We next used monkey platelet-rich plasma (PRP) to study the behavior of cF1232 and GPVI in vitro. Incubation of PRP with CypHer5E-labeled cF1232 at $37^{\circ} \mathrm{C}$ led to a time-dependent increase in fluorescence corresponding to $\mathrm{Ab}$ endocytosis, and little increase in fluorescence was seen when samples were incubated at $4^{\circ} \mathrm{C}$ (Figure 6A). When CypHer5E-labeled cF1232 was incubated at $37^{\circ} \mathrm{C}$ with GPVI-deficient PRP that was obtained from monkeys treated with unlabeled cF1232 for 5 days as described above, CypHer5E fluorescence was not detected in platelets (data not shown), confirming that the in vitro cF1232 internalization and endocytosis was strictly dependent on surface GPVI. However, in contrast to what was seen in vivo, there was no change in surface GPVI expression during incubation at $37^{\circ} \mathrm{C}$ (Figure 6A). 
A
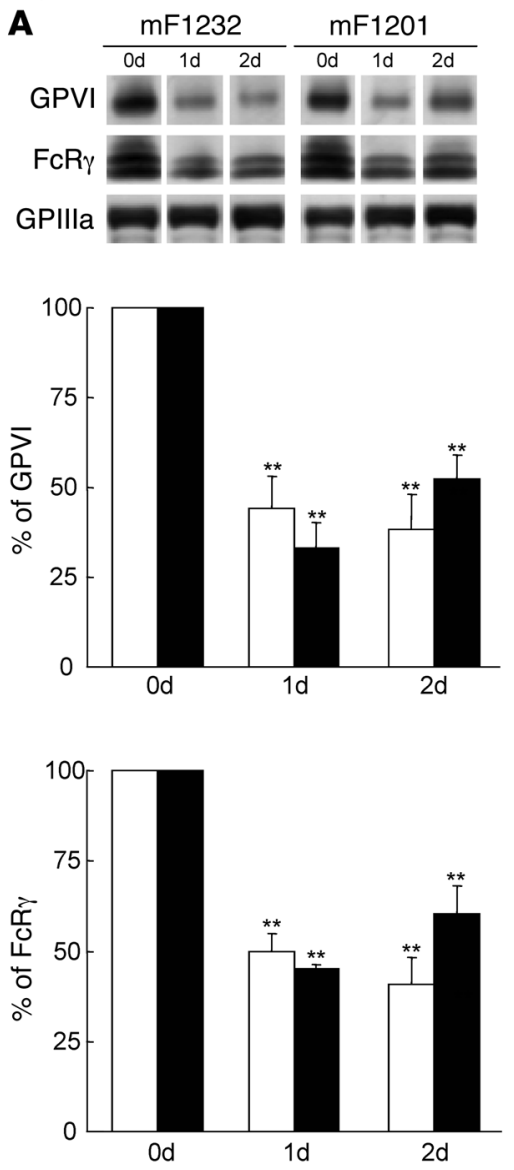

B

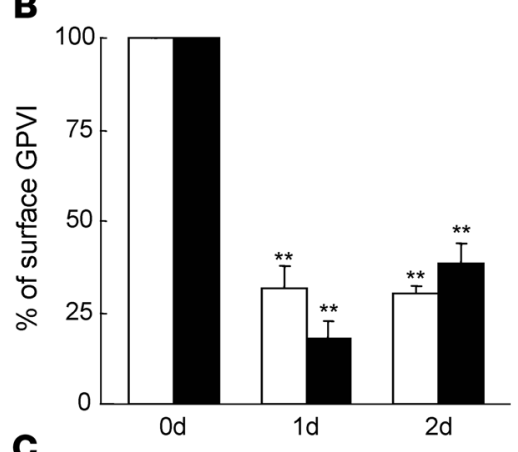

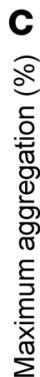

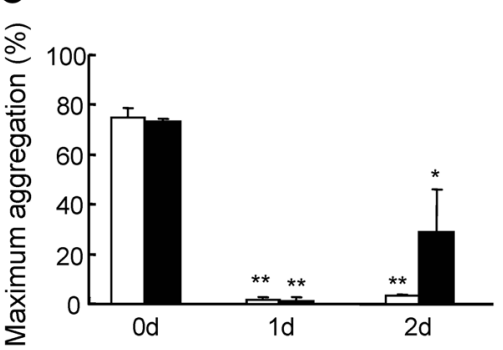

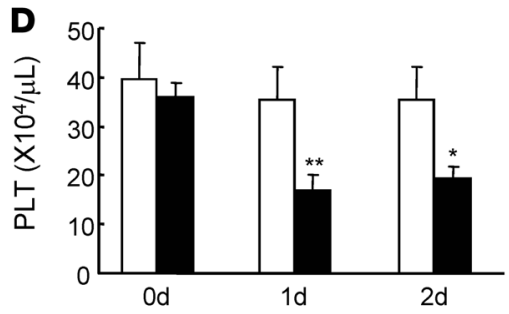

\section{Figure 4}

Effects of mF1232 or mF1201 injected into cynomolgus monkeys on GPVI immunodepletion, collageninduced platelet aggregation, and platelet counts. mF1232 (white bars) or mF1201 (black bars) was i.v. injected at a dose of $0.3 \mathrm{mg} / \mathrm{kg}$ into monkeys. Blood was collected on day 0 (0d), day 1 (1d), and day 2 (2d) after injection. The level of GPVI, FcR $\gamma$, and GPIIla in platelet lysates (A) and the expression of surface GPVI (B) were analyzed by western blotting and by flow cytometry, respectively, using each specific Ab. (A) Representative data of western blotting from 3 separate experiments are shown at the top of the panel. The band density of GPVI (middle panel) or FcR $\gamma$ (bottom panel) was quantified by a Typhoon Scanner 9410 and shown as percentage of that on day 0. (B) Mean fluorescence intensity was normalized to 100 for that on day 0 . Maximal platelet aggregation was monitored using PRP stimulated with $2 \mu \mathrm{g} / \mathrm{ml}$ collagen (C), and platelet (PLT) counts in whole blood (D) were determined. Data are presented as mean \pm SEM of 3 separate experiments. ${ }^{*} P<0.05,{ }^{* *} P<0.01$ versus day 0 .
We next examined whether $\mathrm{cF} 1232$ could be internalized and endocytosed in a platelet activation-independent manner, and PRP was pretreated with $3 \mu \mathrm{M} \mathrm{PGE}_{1}$, a well-known potent inhibitor of platelet activation that acts by elevating intracellular cAMP. In $\mathrm{PGE}_{1}$-treated platelets, as $\mathrm{CF} 1232$ was endocytosed into platelets, surface GPVI expression was lost in parallel and it decreased to approximately $30 \%$ untreated levels after 2 hours of cF1232 treatment (Figure 6B). The same experiment was also performed by replacing $\mathrm{cF} 1232$ with $\mathrm{mF} 1201$. As shown in Figure 6C, mF1201 was endocytosed and significant decrease in surface GPVI expression was observed as with $\mathrm{mF} 1232$. Since we found that $\mathrm{mF} 1201$ induced shedding of surface GPVI in the absence of $\mathrm{PGE}_{1}$ as described in Figure 2D, we performed experiments to compare $\mathrm{Ab}$ endocytosis and the loss of surface GPVI between PGE -treated $_{1}$ and -untreated platelets, and washed monkey platelets were incubated with CypHer5E-labeled or -unlabeled $\mathrm{mAbs}$ in the presence or absence of $\mathrm{PGE}_{1}$ at $37^{\circ} \mathrm{C}$ (Figure 6, D and E). As shown in Figure 6D, both CypHer5E-labeled mF1232 and mF1201 were endocytosed whether or not $\mathrm{PGE}_{1}$ was included in the reaction mixtures. As was seen with PRP, there was a significant decrease in surface GPVI expression in washed platelets incubated with CypHer5Elabeled mF1232 in the presence, but not absence, of $\mathrm{PGE}_{1}$. In contrast, regardless of $\mathrm{PGE}_{1}$ treatment, surface GPVI expression of washed platelets incubated with CypHer5E-labeled mF1201 was significantly decreased. We also examined the supernatants of washed platelet suspensions incubated with either $\mathrm{mAb}$ in

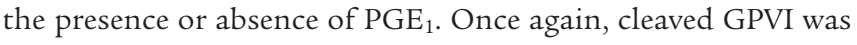

detected by immunoblotting in the supernatants of the samples incubated with mF1201, but not with $\mathrm{mF1232}$, and $\mathrm{PGE}_{1}$ did not significantly affect this (Figure 6E).

We wished to measure an increased amount of soluble GPVI in the plasma from monkeys after mF1232 or mF1201 treatment. For this purpose, we developed a sandwich ELISA assay that could quantitatively detect injected $\mathrm{mAb}$-bound and -unbound soluble GPVI. The increased amounts of soluble GPVI in the plasma from monkeys after mF1232 or mF1201 treatment at a dose of 0.3 $\mathrm{mg} / \mathrm{kg}$ were assessed as described in the Methods and compared between 2 groups. As shown in Figure 7, most of plasma samples from monkeys after mF1201 treatment revealed detectable increase in soluble GPVI content above the limit of ELISA sensitivity until 4 hours after treatment but not thereafter, and the highest levels of increase in soluble GPVI content were observed 1 hour after treatment. In contrast, little increase in soluble GPVI content over the limit of sensitivity was observed in the plasma from monkeys over 24 hours after mF1232 treatment. Statistical analyses revealed that soluble GPVI content in the plasma significantly increased 1 hour after mF1201, but not mF1232, treatment and that the difference of increased amounts of soluble GPVI between those 2 groups was significant. Interestingly, surface GPVI expression levels on platelets from mF1201-treated monkeys appeared to decrease a little more rapidly than those from mF1232-treated monkeys, but those levels did not differ significantly from each other 24 hours after treatment, confirming the finding shown in Figure 4B (Figure 7, top left panel). Furthermore, although the 

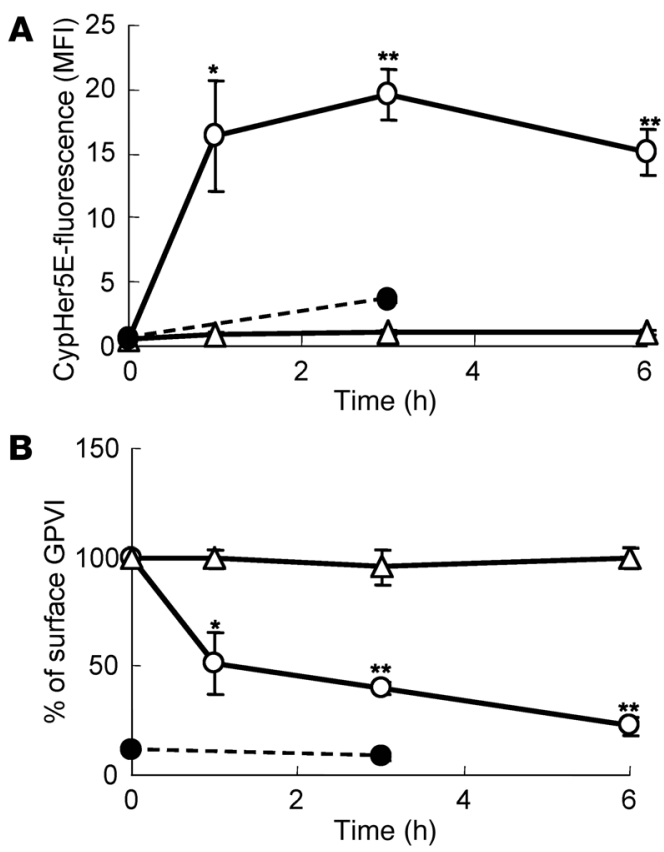

C

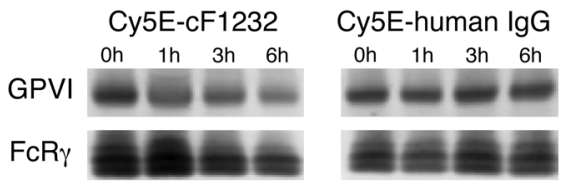

maximal plasma concentration of either injected $\mathrm{mAb}$ was less than $10 \mu \mathrm{g} / \mathrm{ml}$, plasma concentrations of $\mathrm{mF} 1201$ were found to be lower than those of $\mathrm{mF} 1232$ at the indicated time points after injection, suggesting more rapid clearance of mF1201-forming immune complexes with activated platelets/microaggregates or soluble GPVI (Figure 7, top right panel).

In patient YA, sustained anti-GPVI Ab concentrations around $1 \mu \mathrm{g} / \mathrm{ml}$ were sufficient to maintain decreased GPVI surface expression, and we wished to maintain the same concentration of cF1232. Monkeys were injected with a single dose of cF1232 of $1 \mathrm{mg} / \mathrm{kg}$ either i.v. or s.c., and we followed the plasma Ab concentration over time (Figure 8A). Subcutaneous injection of cF1232 led to a sustained plasma concentration over $1 \mu \mathrm{g} / \mathrm{ml}$ for more than 3 days, but i.v. injection achieved this concentration for less than 2 days. Platelets isolated from injected monkeys lost surface GPVI expression within the first day, and this decrease was maintained for 7 to 10 days without significant thrombocytopenia and accompanied unresponsiveness to collagen but not ADP stimulation (Figure 8B and data not shown).

\section{Discussion}

The first patient with platelet GPVI deficiency has been followed up by our department for more than 20 years and has platelets still lacking GPVI, without thrombocytopenia, or any signs of bleeding. Although the patient has been suffering from chronic atrial fibrillation for the last 7 years, she has not received any antithrombotic medications for fear of inducing bleeding events. Additionally, she denies any signs or symptoms of thrombosis in the brain and heart (unpublished observations). The patient's serum IgG, isolated and described in our original report, has been an invaluable tool for

\section{Figure 5}

CypHer5E-labeled cF1232 injected into cynomolgus monkeys is endocytosed into circulating platelets correlating with GPVI depletion. CypHer5E-labeled cF1232 or human IgG was i.v. injected at a dose of $1 \mathrm{mg} / \mathrm{kg}$ into cynomolgus monkeys. The fluorescence intensity (A) in circulating platelets and platelet surface GPVI expression (B) at the indicated time points after injection of CypHer-5E-labeled cF1232 (open circles) or human IgG (open triangles) were analyzed by flow cytometry. The levels of surface GPVI expression are normalized to 100 for day 0 . The filled circles with dotted lines represent monkeys pretreated with $1 \mathrm{mg} / \mathrm{kg}$ unlabeled cF1232. GPVI and FcR $\gamma$ expression in circulating platelets at the indicated time points after injection were analyzed by western blotting (C). All data are presented as mean \pm SEM of 3 separate experiments. ${ }^{*} P<0.05,{ }^{*} P<0.01$ versus control groups at the same time points.

both the immunological detection of GPVI and studies of GPVI function. However, serum isolated more recently is much less reactive and of limited utility. These clinical and laboratory observations hinted to us that the low titer YA-Abs have generated a longterm state of GPVI immunodepletion without serious episodes of bleeding or thrombosis. Then, we characterized YA-Abs over 15 years to identify conditions compatible with long-term GPVI depletion. The concentration of anti-GPVI YA-Abs- 03 was less than 10 $\mu \mathrm{g} / \mathrm{ml}$ and 100 -fold lower than comparable YA-Abs-88, suggesting that YA-Abs-03 might contain anti-GPVI Ab immunodepleting the target in low titer without causing thrombocytopenia.

We generated mouse anti-hGPVI mAbs with the hope of replicating the performance characteristics of YA-Abs. Indeed, 2 representative $\mathrm{mAbs}, \mathrm{mF} 1201$ and $\mathrm{mF} 1232$, were effectively prevented from binding to hGPVI-Fc by YA-Abs. These mAbs did not cross-inhibit one another, and both recognized distinct epitopes on hGPVI. These data also confirm the oligoclonal nature of YA-Abs.

Upon further characterization of $\mathrm{mF} 1201$ and $\mathrm{mF} 1232$, we found striking functional differences between these mAbs tested at the concentrations similar to those of anti-GPVI Abs in the recent plasma of YA, namely at less than $10 \mu \mathrm{g} / \mathrm{ml}$. Treatment of platelets with mF1201 alone resulted in platelet aggregation, activation, and the shedding of surface GPVI. GPVI shedding is thought to be mediated by metalloproteinase-dependent proteolysis, and this may occur in an activation-dependent manner $(20,21)$. In contrast, mF1232 induced neither significant platelet activation such as aggregation and CD62P expression nor the shedding of surface GPVI.

When we mapped the binding sites of mF1201 and of mF1232 using a systematic mutagenesis approach, we demonstrated that mF1201 recognized a previously reported collagen-binding site on hGPVI (26). In contrast, mF1232 recognized what we believe to be a novel epitope on loop 9 (Thr116-Gln122) of the Ig-D2 of hGPVI. It is interesting to consider the contrasting functional data for these $2 \mathrm{mAbs}$ in the context of their binding sites. Treatment of platelets with collagen induces aggregation and activation, and it is likely that the agonistic nature of mF1201 leads to cross-linking of the collagen-binding sites of GPVI. In contrast, mF1232 inhibits collagen-induced aggregation but does not affect CRP-induced aggregation. Interestingly, Dumont et al. reported that Ig-D2 plays a specific and significant role in GPVI binding to collagen but CRP does not. Additionally, Horii et al. determined the crystal structure of the collagen-binding domain of hGPVI and suggested that Ig-D2 of the 2 GPVI molecules formed a parallel, back-to-back dimer on the platelet membrane, matching the dimensions of a collagen fiber 

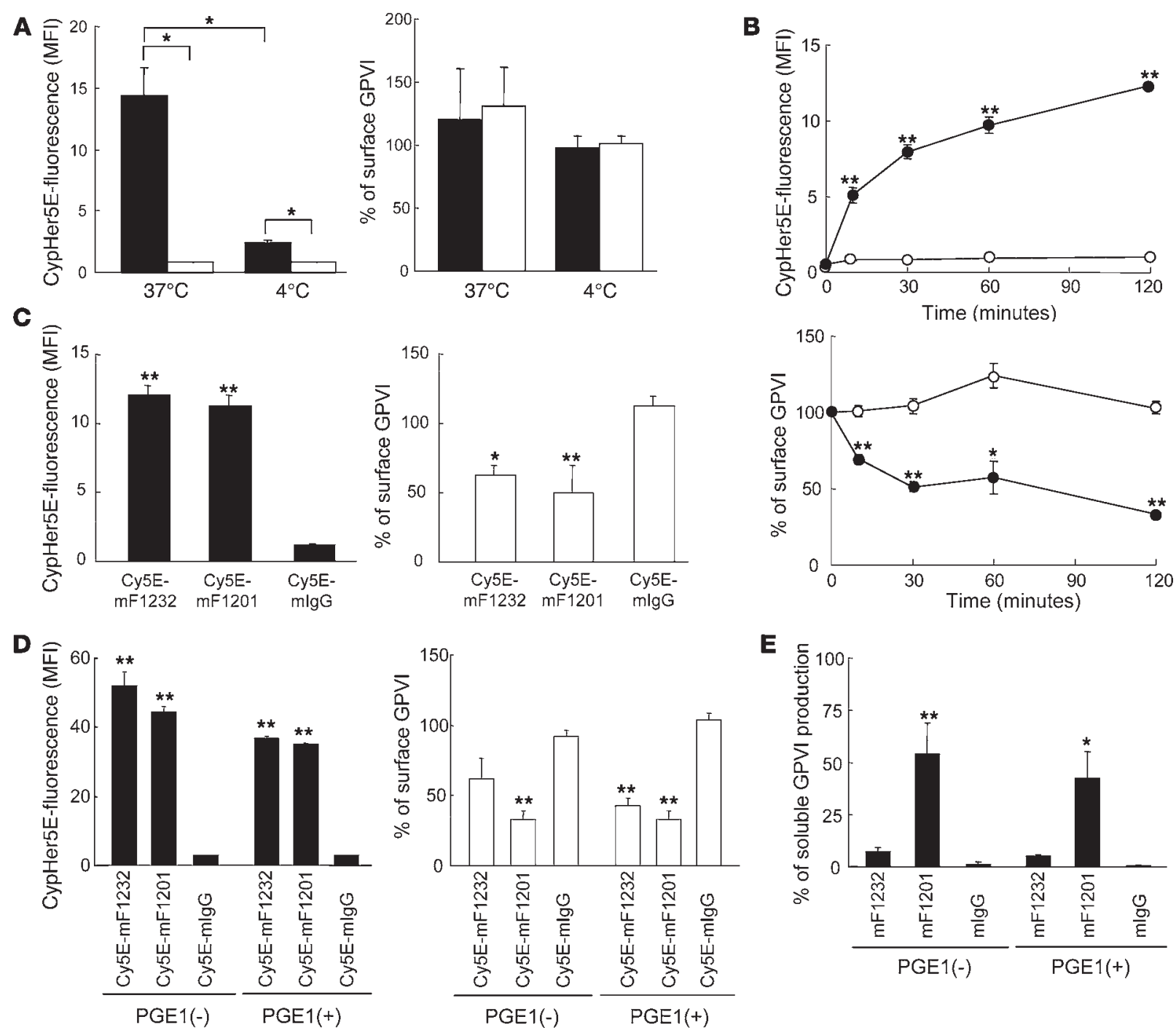

Figure 6

In vitro internalization and endocytosis of GPVI-specific Abs with or without GPVI immunodepletion under various experimental conditions. Cynomolgus PRP was incubated with $10 \mu \mathrm{g} / \mathrm{ml}$ CypHer5E-labeled (Cy5E-labeled) Ab in vitro under various experimental conditions as follows: 60 minutes incubation with cF1232 (black bars) or human IgG (white bars) at $37^{\circ} \mathrm{C}$ or $4^{\circ} \mathrm{C}(\mathrm{A})$, incubation with cF1232 (filled circles) or human IgG (open circles) for up to 120 minutes at $37^{\circ} \mathrm{C}$ in the presence of $3 \mu \mathrm{M} \mathrm{PGE}$ (B), 60 minutes incubation with mF1232, $\mathrm{mF1201,} \mathrm{or} \mathrm{mouse} \mathrm{IgG}$ ( $\mathrm{mlgG}$ ) at $37^{\circ} \mathrm{C}$ in the presence of $3 \mu \mathrm{M} \mathrm{PGE}(\mathbf{C})$. Washed cynomolgus platelets were treated with the indicated Abs in the absence or presence of $3 \mu \mathrm{M}$ PGE $E_{1}$ for 60 minutes at $37^{\circ} \mathrm{C}$ (D). (A-D) The fluorescence in platelets (left panels) and surface GPVI expression (right panels) were analyzed by flow cytometry. Surface GPVI expression before incubation was normalized to 100. (E) Washed cynomolgus platelets were treated with $0.15 \mu \mathrm{g} / \mathrm{ml}$ convulxin, $10 \mu \mathrm{g} / \mathrm{ml} \mathrm{mF1232,} 10 \mu \mathrm{g} / \mathrm{ml} \mathrm{mF1232}$, or $10 \mu \mathrm{g} / \mathrm{ml}$ mouse IgG for 60 minutes at $37^{\circ} \mathrm{C}$ in the absence or presence of $\mathrm{PGE}_{1}$ and sedimented to separate supernatants. The supernatants were analyzed by western blotting with rabbit anti-human GPVI polyclonal $\mathrm{Ab}$. The band density corresponding to soluble GPVI was quantified by a Typhoon Scanner 9410 , and the value for convulxin treatment in the absence of $\mathrm{PGE}_{1}$ was normalized to 100 . All results represent mean $\pm \mathrm{SEM}$ of $3(\mathbf{A}, \mathbf{D}$, and $\mathbf{E})$ or $4(\mathbf{B}$ and $\mathbf{C})$ separate experiments. ${ }^{*} P<0.05$, ${ }^{* *} P<0.01$ versus control groups.

(32). Taken together with these reports, it is tempting to speculate the following mechanisms by which mF1232 binding to loop 9 of Ig-D2 interferes with collagen-mediated, but not CRP-mediated, platelet interaction. Loop 9 of Ig-D2 might contain a heretofore undefined collagen-binding site but not a CRP-binding site. If the site of $\mathrm{mF} 1232$ binding in loop 9 does not participate in the back-toback dimer formation described by Horii et al. (32), mF1232 binding to GPVI might be able to disrupt the adoption of the appropriate dimer structure. Because CRP binds monomeric GPVI, its ability to induce platelet activation might be unaffected by mF1232. These reports and our present findings suggest a novel series of experiments to better clarify the mechanism of collagen and CRP binding to GPVI that should be undertaken in the future.

The generation of $2 \mathrm{mAbs}$ that bind GPVI with distinct effects on platelet activation allowed us to further investigate the mechanisms underlying Ab-induced GPVI immunodepletion using cynomolgus monkeys as a model system. First, we showed that both mAbs bound cGPVI, a protein highly homologous to hGPVI, 

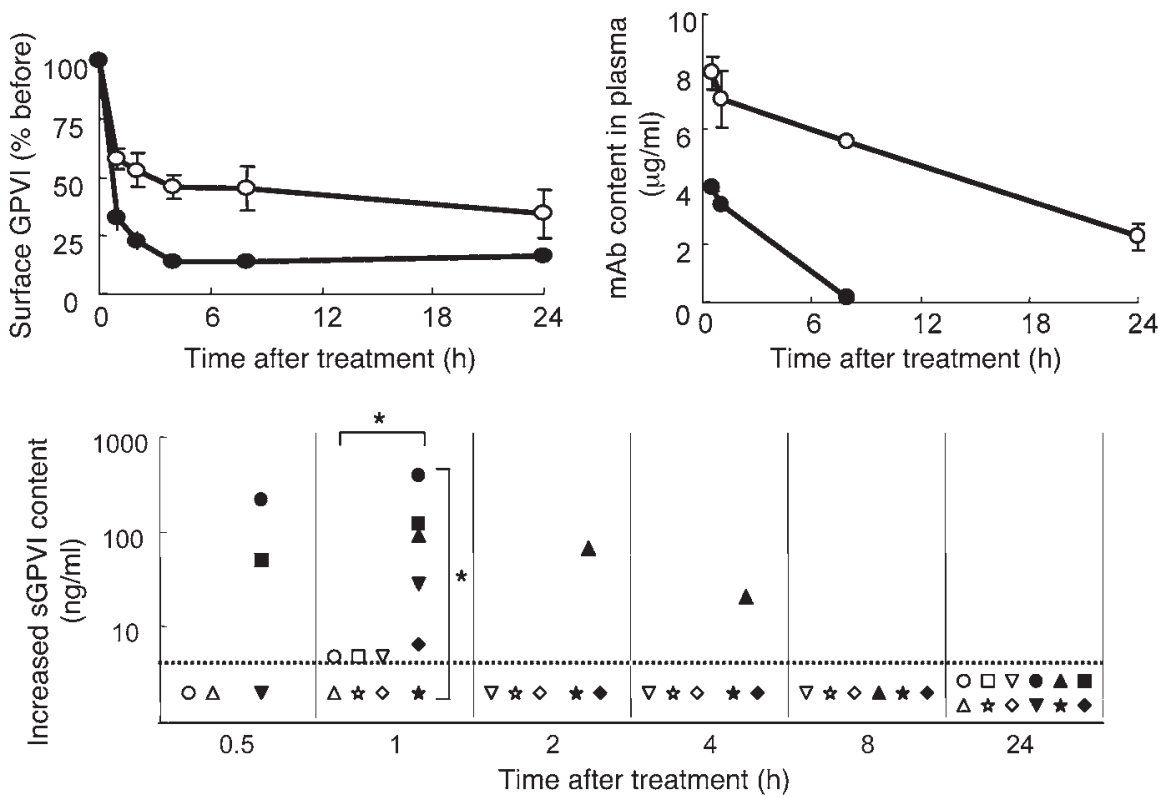

Figure 7

Comparison of increased amounts of soluble GPVI in the plasma from monkeys after F1232 or F1201 treatment. mF1232 (open symbols) or mF1201 (filled symbols) was i.v. injected at a dose of $0.3 \mathrm{mg} / \mathrm{kg}$ into monkeys. Blood was collected before injection and at the indicated time points after injection. The increased amounts of soluble GPVI in the plasma at the indicated time points after each treatment (bottom panel), the expression of surface GPVI (top left panel), and plasma concentrations of each mAb (top right panel) were assessed as described in the Methods and compared between 2 groups. The number of tested monkeys ranged from 2 to 6 at each time point. Data are expressed as the amount of cGPVI-Fc, a standard, or as mean \pm SEM. The dotted bar represents the limit of sensitivity of the assay $(4.9 \mathrm{ng} / \mathrm{ml})$. ${ }^{*} P<0.05$ versus $0 \mathrm{ng} / \mathrm{ml}$ or $\mathrm{mF} 1232$ versus $\mathrm{mF} 1201$. and they affected platelet function in monkeys in a manner similar to that seen for humans. Notably, both mAbs induced GPVI immunodepletion when i.v. injected into monkeys at a low dose of $0.3 \mathrm{mg} / \mathrm{kg}$. However, injection of mF1201 caused significant thrombocytopenia that might reflect shortened platelet life span. Most of previous studies provided evidence for the metalloproteinase-dependent cleavage of surface GPVI both in vitro and in vivo $(20,21,33,34)$. We clearly showed that $10 \mu \mathrm{g} / \mathrm{ml} \mathrm{mF} 1201$, but not mF1232, induced shedding of surface GPVI in vitro, and it is likely that mF1201-induced shedding is at least partly responsible for the reductions in GPVI surface expression seen in vivo. However, there was no shedding seen in vitro following $\mathrm{mF} 1232$ treatment, but GPVI was rapidly lost from the platelet surface following mF1232 injection. This suggested that intracellular degradation might be responsible for reductions in GPVI expression following mF1232 treatment.

We wished to obtain direct evidence for the internalization and endocytosis of GPVI using a fluorescent endocytosis probe, CypHer5E, which becomes fluorescent in the acidic environment of intracellular organelles such as endosomes and lysosomes (29, 30 ). We also used a chimeric Ab based on mF1232 (cF1232) for these studies. The behavior of cF1232 toward both human and cynomolgus platelets was the same as mF1232. Importantly, cF1232 did not activate human platelets in vitro, even at a maximal test concentration of $3 \mathrm{mg} / \mathrm{ml}$. Therefore, it is very likely that
cF1232 binding to loop 9 of Ig-D2 on GPVI does not result in GPVI-mediated activation of human platelets, not only in vitro but also when injecting cF1232 into humans. When CypHer5E-labeled cF1232 was injected into monkeys, there was a dramatic GPVIdependent increase in fluorescence in circulating platelets and there was a corresponding decrease in surface GPVI expression. These data indicate that injected cF1232 bound to surface GPVI is internalized and endocytosed into circulating platelets. A similar surface GPVI-dependent endocytosis of cF1232 was also observed when platelets were incubated with CypHer5E-labeled cF1232 at $37^{\circ} \mathrm{C}$ but not $4^{\circ} \mathrm{C}$ in vitro. However, in contrast to the results seen in vivo, there was sustained GPVI expression at the platelet surface. These results suggest that in vitro GPVI was internalized and rapidly recycled back to the platelet surface without degradation in order to maintain surface GPVI level, but an unknown mechanism was operating to downregulate surface GPVI level in vivo.

The solution came from the experiment to see whether in vitro internalization and endocytosis of CypHer5E-labeled cF1232 into platelets could occur when a cAMP-elevating agent, $\mathrm{PGE}_{1}$, was included in the reaction mixture. In the presence of $\mathrm{PGE}_{1}, \mathrm{cF} 1232$ was endocytosed, and there was a corresponding reduction in surface GPVI that was not shed in vitro. In vivo, the vasculature produces prostacyclin, a potent endogenous inhibitor of platelet activation that increases intracellular cAMP levels analogous to $\mathrm{PGE}_{1}$. It is very likely that prostacyclin constitutively released from the vas-

\section{Figure 8}

Pharmacokinetics and long-term antiplatelet effects after cF1232-treatment. (A) cF1232 was i.v. (filled circles) or s.c. (open circles) injected at a dose of $1 \mathrm{mg} / \mathrm{kg}$ into cynomolgus monkeys, and plasma concentrations of cF1232 were measured by ELISA. (B) After s.c. injection of cF1232, maximal platelet aggregation in response to $2 \mu \mathrm{g} / \mathrm{ml}$ collagen (filled circles) or $20 \mu \mathrm{M}$ ADP (filled squares) was periodically monitored. The aggregation rate was normalized to $100 \%$ for those on day 0 . The experiments were carried out with 3 monkeys and the data represent mean \pm SEM.
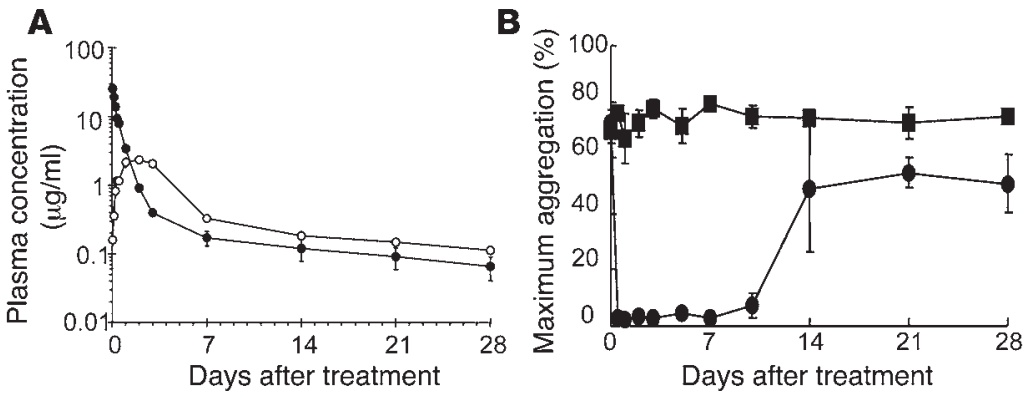
culature is essential for the observed downregulation of GPVI seen in vivo, and we must recreate this in vivo effect to observe a similar phenomena in vitro. Importantly, we found a statistically significant increase in plasma concentrations of soluble GPVI in mF1201treated, but not mF1232-treated, monkeys and also a significant difference of increase in those between 2 groups. Taken together, this is what we believe to be the first direct evidence demonstrating specific Ab-induced GPVI internalization and endocytosis both in vitro and in vivo, and it resolves the questions regarding acquired GPVI deficiency caused by specific auto-Abs $(12,19)$. Interestingly, $\mathrm{mF} 1201$-induced proteolytic shedding occurred even in the presence of $\mathrm{PGE}_{1}$, indicating that the elevation of intracellular cAMP cannot completely inhibit the proteolytic shedding. Nevertheless, mF1201 was endocytosed to the same extent as cF1232 or mF1232 during incubation at $37^{\circ} \mathrm{C}$ in vitro, suggesting that $\mathrm{mF} 1201$ could deplete surface GPVI through both proteolytic shedding and endocytosis. With regard to receptor internalization, Nieswandt et al. showed that decreased surface GPVI expression preceded reductions in GPVI in whole platelet lysates within 24 hours after injection of the anti-mGPVI mAb JAQ-1 into WT mice (14). However, the presence of a large, internal pool of GPVI has been proven (23) and could explain their findings. Indeed, they confirmed that JAQ-1 caused proteolytic shedding of surface GPVI that was associated with the occurrence of thrombocytopenia (34). In contrast to what we directly demonstrated here using CypHer5E-labeled mAbs and WT platelets or monkeys both in vivo and in vitro, they used biotinlabeled JAQ-1 and mutant mice lacking GPVI downstream signaling molecules to show JAQ-1-induced GPVI internalization in vivo but not in vitro (34). Without using such mutant animals, we definitely showed that the Ig-D2-specific mAbs were endocytosed into platelets irrespective of GPVI downregulation in a surface GPVIdependent manner but caused GPVI internalization and endocytosis without significant GPVI shedding both in vivo and in vitro when platelets were also exposed to a cAMP-elevating agent. Taken together with our previous report that GPVI downstream signals are cAMP-insensitive (7), the mechanisms underlying specific $\mathrm{Ab}$ induced depletion of surface GPVI through internalization and endocytosis could not be simply explained by GPVI downstream signals themselves. It was of great interest that GPVI immunodepletion could be safely induced without thrombocytopenia following injection of mF1232 into WT monkeys (22). In view of the recent report that JAQ-1 treatment caused significant thrombocytopenia in WT mice but did not in the mutant mice, revealing no shedding of surface GPVI (34), it is noteworthy that injection of mAbs recognizing what we believe to be a novel epitope on Ig-D2 of hGPVI did not cause significant thrombocytopenia in WT monkeys as shown here. Clearly, anti-GPVI mAbs such as mF1201 that strongly cause thrombocytopenia are unsuitable for clinical application.

In a clinical application of $\mathrm{cF} 1232$, its injection via s.c. route could be more practical and useful than that via i.v. route to maintain a longer effect of GPVI immunodepletion. In fact, we found that a single s.c. injection of cF1232 into monkeys led to sustained low titer plasma concentrations and long-term antiplatelet effects due to GPVI immunodepletion without significant thrombocytopenia. Present work warrants further studies to emphasize the therapeutic promise of the $\mathrm{cF} 1232$ initially characterized here.

\section{Methods}

Reagents. CRP was synthesized as described previously (35). cDNA of hGPVI was prepared as described previously (5). Rabbit anti-GPVI polyclonal Ab was produced by immunizing rabbits with a synthetic peptide corresponding to amino acids Glu148-Arg162 of hGPVI. CypHer5E mono NHS ester and R-PE Labeling Kit- $\mathrm{NH}_{2}$ were purchased from GE Healthcare and Dojindo Laboratories, respectively. Conjugation of Abs with CypHer5E or PE was performed according to the manufacturer's instructions. All other reagents were obtained as previously reported $(6-8,10,17,23,25)$.

Recombinant GPVI proteins and mutant proteins. The cDNA encoding the extracellular region of hGPVI (Met-1 of the signal peptide to Asn-269) and the $\mathrm{Fc}$ regions of mouse $\operatorname{IgG}_{2 \mathrm{a}}$ or human $\mathrm{IgG}_{1} \mathrm{H}$ chain were cloned by genomic PCR, and these fragments were inserted to downstream of the human elongation promoter in the pEF-expression vector (36). An expression plasmid encoding a mutant-hGPVI protein was constructed using site-specific mutagenesis by overlap extension with mGPVI as a template (37). The cDNA encoding CGPVI was cloned by PCR using primer pairs based on the hGPVI cDNA and cynomolgus monkey genomic DNA as a template. The cDNA encoding the extracellular region of mGPVI was cloned by genomic PCR using primer pairs based on the mGPVI cDNA sequence submitted to GENBANK with accession number XM145298. The cDNA encoding mutated GPVI was cloned into the pEF-expression vector to allow fusion with the human $\mathrm{Fc}$ region. COS- 1 or $\mathrm{CHO}$ cells were transfected with expression plasmids using FuGENE6 (Roche) according to the manufacturer's instructions and cultured in serum-free medium in $5 \%$ $\mathrm{CO}_{2}$ at $37^{\circ} \mathrm{C}$. Recombinant GPVI proteins fused to human Fc or mouse Fc regions were purified from culture supernatants by protein A-Sepharose affinity chromatography (Prosep-A; Millipore). The extracellular domain of cGPVI or mGPVI was also fused to the human Fc region and each fusion protein was prepared as described above. The nucleotide sequence of cGPVI has been submitted to the DDBJ/EMBL/GenBank databases with accession number AB292416 (http://www.ddbj.nig.ac.jp).

Generation of mouse anti-GPVI mAbs. Female ddY mice (Japan SLC Inc.) at 8 weeks of age were primed and boosted at 11-day intervals with $20 \mu \mathrm{g}$ of the recombinant extracellular domain of hGPVI fused to the Fc region of mouse $\operatorname{IgG}_{2 \mathrm{a}}$ with adjuvant. Three days later, lymphocytes were harvested from lymph nodes and fused with mouse myeloma cells (P3U1; ATCC) by the polyethylene glycol method. Hybridomas secreting anti-GPVI mAbs were identified by ELISA using hGPVI-Fc-coated plates. Mouse anti-GPVI mAbs were purified by Prosep-A column. In some experiments, those mAbs were conjugated with peroxidase by a periodate method (38).

Construction of human/mouse chimera anti-GPVI $m A b$. Single-stranded cDNAs were prepared from the hybridoma secreting mouse anti-GPVI $\mathrm{mAb}$ using the SuperScript III First-Strand Synthesis System for RT-PCR kit (Invitrogen). The Ig-variable region was amplified by a PCR using the obtained single-stranded cDNA as a template and Mouse Ig-Primer Set (Merck). The extreme $3^{\prime}$-primer was designed with a restriction site allowing fusion with human $\mathrm{H}$ chain $\mathrm{C} \gamma 4 \mathrm{cDNA}$. Using these primers, $\mathrm{PCR}$ was performed, amplifying both the $\mathrm{H}$ - and $\mathrm{L}$-chain variable regions followed by subcloning into the $\mathrm{pT} 7 \mathrm{BlueT}$ vector (Merck). The gene fragment encoding $\mathrm{H}$ chain ligated to $\mathrm{Cg} 4 \mathrm{cDNA}$ and encoding $\mathrm{L}$ chain were introduced into appropriate pEF-expression plasmids. COS-1 cells or $\mathrm{CHO}$ cells were transfected with the expression plasmids using FuGENE6, and cells were cultured in serum-free medium in $5 \% \mathrm{CO}_{2}$ at $37^{\circ} \mathrm{C}$. Human/mouse chimera anti-GPVI $\mathrm{mAb}$ was purified from the supernatant by protein Acolumn affinity chromatography.

Quantification of anti-GPVI Abs in the patient plasma. hGPVI-Fc was biotinylated with D-biotinoyl- $\varepsilon$-aminocaproic acid- $N$-hydroxysuccinimide ester (Roche) according to the manufacturer's instructions. Sample plasma was obtained from patient YA and stored at $-70^{\circ} \mathrm{C}$ during our previously reported studies $(6-8,10,17,23)$. Fifty microliters of appropriately diluted plasma or control solutions, containing known amounts of a human/ mouse chimera anti-GPVI mAb, were incubated with $50 \mu \mathrm{l}$ of biotinylated 
hGPVI-Fc $(2 \mu \mathrm{g} / \mathrm{ml})$ for 2 hours at $37^{\circ} \mathrm{C}$. The mixture was then applied to a 96-well plate (Nunc-Immuno Module C8 Maxisorp; Nunc) coated with streptavidin $(10 \mu \mathrm{g} / \mathrm{ml})$. After 20 -minutes incubation at $37^{\circ} \mathrm{C}$, the wells were washed 5 times with saline containing $0.05 \%$ Tween 20 . The bound Abs were colorimetrically quantified using peroxidase-labeled anti-human kappa and lambda chain Abs (Dako) and tetramethyl benzidine (TMB) (TMB 1 Component HRP Microwell Substrate; BioFX Laboratories Inc.).

Cross-reactivities of YA-Abs with mouse anti-GPVI $m A$ s sor the binding to hGPVI-Fc. Sample plasma from patient YA was applied on a Prosep-A column equilibrated with D-PBS. The IgG fraction was eluted with $0.1 \mathrm{M}$ Glycine- $\mathrm{HCl}(\mathrm{pH} 3.0)$ and neutralized in $1 \mathrm{M}$ Tris- $\mathrm{HCl}(\mathrm{pH}$ 8.5), and this was subsequently applied onto the hGPVI-Fc column prepared with formyl-cellulofine (Seikagaku Corporation) according to the manufacturer's instructions. Anti-GPVI Abs were eluted with $10 \mathrm{mM} \mathrm{HCl}$ and neutralized with 10x D-PBS. The concentration of anti-GPVI Abs was determined by ELISA as described above.

Fifty microliters of the serially diluted anti-GPVI Abs was applied to a 96-well plate (Nunc-Immuno Module C8 Maxisorp; Nunc) coated with 0.5 $\mu \mathrm{g} / \mathrm{ml} \mathrm{hGPVI-Fc}$ and incubated overnight at $4^{\circ} \mathrm{C}$. Fifty microliters of a peroxidase-labeled anti-GPVI $\mathrm{mAb}(50 \mathrm{ng} / \mathrm{ml})$ was then added to each well and incubated for 45 minutes at $37^{\circ} \mathrm{C}$. The bound peroxidase-labeled mAbs were colorimetrically quantified by reaction with TMB substrate.

Binding of anti-GPVI mAbs to WT and mutant GPVI-Fc. Two types of ELISA were conducted. First, $25 \mu \mathrm{l}$ of $50 \mathrm{ng} / \mathrm{ml}$ peroxidase-labeled anti-GPVI mAb and $1 \mu \mathrm{g} / \mathrm{ml} \mathrm{WT}$, mutant GPVI-Fc, or a control buffer were added to wells of a 96-well plate coated with hGPVI-Fc and incubated for 1 hour at $37^{\circ} \mathrm{C}$. After incubation, the bound peroxidase-labeled mAbs were quantified by ELISA. Additionally, serially diluted anti-GPVI mAbs were added to a 96-well plate coated with WT or mutant GPVI-Fc. After 1 hour incubation at $37^{\circ} \mathrm{C}$, the bound $\mathrm{mAbs}$ were colorimetrically quantified by reaction with peroxidase-labeled anti-mouse Igs (Dako) and TMB substrate.

Blood sample preparation. Venous blood was collected from the patient and healthy volunteers after obtaining informed consent, and blood was collected from cynomolgus monkeys after approval by the ethics committees at Kyoto University and Mochida Pharmaceutical Co. Ltd. Anticoagulation of blood with $0.1 \mathrm{vol}$ of $3.8 \%$ sodium citrate and preparation of PRP, platelet-poor plasma, or washed platelets were performed as described previously $(12,39)$.

Platelet aggregation. The ability of anti-GPVI mAbs to activate platelets was tested by incubating PRP $\left(3 \times 10^{5}\right.$ platelets $\left./ \mu \mathrm{l}\right)$ with $1-10 \mu \mathrm{g} / \mathrm{ml} \mathrm{mAbs}$ under stirring conditions at $208 \mathrm{~g}$ in an aggregometer (MCM Hema Tracer 313M; MC Medical). The ability of anti-GPVI mAbs to inhibit platelet activation was examined by preincubating PRP with or without $10 \mu \mathrm{g} / \mathrm{ml}$ $\mathrm{mAbs}$ for 8 minutes followed by stimulation with either $1 \mu \mathrm{g} / \mathrm{ml}$ collagen, $4 \mu \mathrm{g} / \mathrm{ml} \mathrm{CRP}, 5 \mu \mathrm{M}$ ADP, $1.5 \mathrm{mg} / \mathrm{ml}$ ristocetin, or $50 \mu \mathrm{M}$ TRAP. Maximal platelet aggregation was monitored over 12 minutes.

In vitro CD62P expression assay. PRP $\left(3 \times 10^{5}\right.$ platelets/ $\left.\mu \mathrm{l}\right)$ was incubated with $1-10 \mu \mathrm{g} / \mathrm{ml}$ anti-GPVI mAbs or $1.5 \mu \mathrm{g} / \mathrm{ml} \mathrm{CRP} \mathrm{as} \mathrm{a} \mathrm{positive} \mathrm{control} \mathrm{for}$ 30 minutes at $37^{\circ} \mathrm{C}$. Incubated samples were fixed with $1 \%$ paraformaldehyde for 30 minutes at $4^{\circ} \mathrm{C}$ and washed 2 times with D-PBS washing buffer (D-PBS, 2.5\% heat inactivated FBS, $2.5 \mathrm{mM}$ EDTA). CD62P expression on platelets was determined by flow cytometry.

In vitro shedding assay. In vitro shedding assay was performed as described previously with slight modifications $(20,21)$. Washed platelets $\left(3 \times 10^{8}\right.$ platelets/ $\mu \mathrm{l})$, suspended in Tyrode-HEPES buffer $(137 \mathrm{mM} \mathrm{NaCl}, 2.7 \mathrm{mM}$ $\mathrm{KCl}, 3 \mathrm{mM} \mathrm{NaH} \mathrm{PO}_{4}, 10 \mathrm{mM}$ HEPES, $5.5 \mathrm{mM}$ glucose, $\mathrm{pH}$ 7.4), were pretreated with or without $3 \mu \mathrm{M} \mathrm{PGE}_{1}$ for 10 minutes at $37^{\circ} \mathrm{C}$ before incubation with anti-GPVI $\mathrm{mAbs}$ or convulxin $(0.15 \mu \mathrm{g} / \mathrm{ml})$ as a positive control for 60 minutes at $37^{\circ} \mathrm{C}$. The reaction was stopped by the addition of one-fifth vol of $25 \mathrm{mM}$ EDTA, containing $6 \times$ complete protease inhibitor cocktail
(Complete, EDTA free; Roche). Platelets were sedimented by centrifugation at $15,000 \times \mathrm{g}$ for 1 minute to separate the platelet pellets from supernatant. The platelet pellets and supernatant were analyzed by western blotting.

In vivo experiments. All experiments using cynomolgus monkeys were approved by the animal ethics committee at Mochida Pharmaceutical Co. Ltd. Anti-GPVI mAbs, CypHer5E-labeled or -unlabeled anti-GPVI mAbs, or human IgG were i.v. injected into male monkeys $(2.5 \sim 4.5 \mathrm{~kg}$, from HAMRI Co. Ltd.). Citrated blood was collected at time periods indicated in the legends for Figures 4, 5, 7, and 8, and the platelet count was determined by a hematology analyzer (F-820; Sysmex).

Assessment of the increased amounts of soluble GPVI in the plasma from monkeys after anti-GPVI $m A b$ treatment. Either the plasma from $\mathrm{mF} 1232$-treated monkeys or control plasma containing known amounts of cGPVI-Fc as a standard was mixed with $50 \mu \mathrm{g} / \mathrm{ml} \mathrm{mF1232} \mathrm{and} \mathrm{diluted} \mathrm{5-fold} \mathrm{with} \mathrm{PBS}$ containing $0.05 \%$ Tween 20 and $0.1 \%$ BSA. These mixtures were transferred to a 96-well plate coated with a human/mouse chimera anti-hGPVI mAb, cF1232-10-1, that did not cross-react with $\mathrm{mF} 1232$. After incubation overnight at $4^{\circ} \mathrm{C}$, peroxidase-labeled anti-mouse IgG Fc Ab (Bethyl) was added, followed by incubation for 90 minutes at $37^{\circ} \mathrm{C}$. The bound was colorimetrically detected by a reaction with TMB substrate. When dealing with the plasma from $\mathrm{mF} 1201$-treated monkeys, the same procedure was also performed by replacing mF1232 and cF1232-10-1 with mF1201 and cF123218-3, respectively. Since the plasma before treatment was equivalent to control plasma with respect to undetectable soluble GPVI content and both were diluted 5-fold for the assay, potential trace amounts of soluble GPVI in those plasmas, if any, were considered almost equally negligible. Therefore, the levels of soluble GPVI content in both the plasma before treatment and control plasma were assumed to be $0 \mathrm{ng} / \mathrm{ml}$, and the limit of sensitivity of the assay for nondiluted plasma samples was determined to be $4.9 \mathrm{ng} / \mathrm{ml}$ of cGPVI-Fc. In order to assess increased amounts of soluble GPVI in the plasma after treatment, optical densities of diluted plasma before treatment and control plasma were subtracted from the values of diluted plasma after treatment and control plasma containing cGPVI-Fc, respectively.

Measurement of plasma concentrations of anti-GPVI mAb. Appropriately diluted plasma or control plasma containing known amounts of antiGPVI mAb was added to a 96-well plate coated with hGPVI-Fc. After 1 hour incubation at $37^{\circ} \mathrm{C}$, the bound $\mathrm{mAbs}$ were colorimetrically quantified by the reaction with peroxidase-labeled anti-mouse or -human kappa (Dako) and TMB substrate.

In vitro internalization and endocytosis assay. Washed platelets or PRP were pretreated with or without $3 \mu \mathrm{M} \mathrm{PGE}_{1}$ for 10 minutes at $37^{\circ} \mathrm{C}$ before incubation with $10 \mu \mathrm{g} / \mathrm{ml} \mathrm{CypHer5E-labeled} \mathrm{mAbs}$ at $37^{\circ} \mathrm{C}$ or on ice for the time periods indicated in the legend for Figure 6 . After incubation, surface GPVI expression and the fluorescence derived from the endocytosed CypHer5E were determined by flow cytometry.

Western blot analysis. The platelet pellets, the supernatants, or the washed platelet suspensions, prepared as described above, were lysed in the SDS sample buffer containing 2-mercaptoethanol and boiled for 5 minutes. Samples were subjected to SDS-PAGE using 5\%-20\% gradient Tris- $\mathrm{HCl}$ gels (ATTO), electrotransferred to PVDF membranes (Millipore), and immunoblotted with rabbit anti-human GPIIIa polyclonal Abs (Santa Cruz) or anti-GPVI polyclonal Abs. For the detection of $F c R \gamma$, samples were subjected to SDS-PAGE using 10\%-20\% gradient tricine gels (Invitrogen) and immunoblotted with rabbit anti-human FceRI $\gamma$-subunit polyclonal Abs (Millipore). All blots were probed with HRP-conjugated goat anti-rabbit Abs (Dako), developed with ECL-Plus (GE Healthcare), and imaged using a Typhoon Scanner 9410 (GE Healthcare). The band density was quantified by the analysis software ImageQuant 5.2 (GE Healthcare).

Flow cytometry. Washed platelet suspensions or PRP were diluted to a final concentration of $1 \times 10^{4}$ or $3 \times 10^{4}$ platelets $/ \mu$ with D-PBS washing buffer. 
Diluted samples were then divided into 2 parts. The first part was diluted more than 25-fold with HEPES buffer ( $\mathrm{pH} 8.0$ ) containing $2.5 \mathrm{mM}$ EDTA and analyzed in a CYTOMICS FC500 flow cytometer (Beckman Coulter) for quantification of the endocytosed CypHer5E. The second part was incubated for 30 minutes on ice with saturating amounts of a PE-conjugated anti-GPVI $\mathrm{mAb}$, which showed no competition with an anti-GPVI $\mathrm{mAb}$ present in the sample or an isotype-matched negative control $\mathrm{Ab}$, diluted, and analyzed in a flow cytometer for quantification of platelet surface GPVI. Quantification of platelet CD62P was performed with a PE-conjugated anti-human CD62P $\mathrm{Ab}$ (Beckman Coulter) under similar conditions as described above. In some experiments, fluorescence intensities were expressed as mean fluorescence intensity (MFI), from which the value of MFI of control was subtracted. All data were analyzed with FlowJo software (Tree Star Inc.).

Statistics. Comparisons between 2 groups and also those among more than 2 groups were made with a Student's unpaired or paired $t$ test and with a Dunnett test, respectively. When analyzing the data, including the values below the limit of sensitivity of the assay, the appropriate nonparametric (Wilcoxon, Mann-Whitney) test was performed. A value of $P<0.05$ was considered statistically significant. The analyses were performed with the StatLight statistical software (Yukms Co. Ltd.).

\section{Acknowledgments}

Authors thank Yumi Hotta, Kotarou Hoshida, Keiko Hoshida, and Shin Kumadaki for the preparation of chimera Ab and Masayuki Ichioka for the technical assistance with flowcytometric analysis, western blot analysis, and epitope analysis. We also thank Tarou Seo for helping with animal study and maintenance of the animals.

Received for publication April 26, 2007, and accepted in revised form February 13, 2008.

Address correspondence to: Hiroshi Takayama, Department of Health and Nutrition, School of Human Cultures, The University of Shiga Prefecture, 2500 Hassakacho, Hikone, Shiga 522-8533, Japan. Phone: 81-749-28-8441; Fax: 81-749-28-8519; E-mail: htakayam@shc.usp.ac.jp.
1. Bhatt, D.L., and Topol, E.J. 2003. Scientific and therapeutic advances in antiplatelet therapy. Nat. Rev. Drug Discov. 2:15-28.

2. Jackson, S.P., and Schoenwaelder, S.M. 2003. Antiplatelet therapy: in search of the 'magic bullet'. Nat. Rev. Drug Discov. 2:775-789.

3. Clemetson, J.M., Polgar, J., Magnenat, E., Wells, T.N., and Clemetson, K.J. 1999. The platelet collagen receptor glycoprotein VI is a member of the immunoglobulin superfamily closely related to $\mathrm{Fc} \alpha \mathrm{R}$ and the natural killer receptors. J. Biol. Chem. 274:29019-29024.

4. Jandrot-Perrus, M., et al. 2000. Cloning, characterization, and functional studies of human and mouse glycoprotein VI: a platelet-specific collagen receptor from the immunoglobulin superfamily. Blood. 96:1798-1807.

5. Ezumi, Y., Uchiyama, T., and Takayama, H. 2000. Molecular cloning, genomic structure, chromosomal localization, and alternative splice forms of the platelet collagen receptor glycoprotein VI. Biochem. Biophys. Res. Commun. 277:27-36.

6. Tsuji, M., Ezumi, Y., Arai, M., and Takayama, H. 1997. A novel association of $F c$ receptor $\gamma$-chain with glycoprotein VI and their co-expression as a collagen receptor in human platelets. J. Biol. Chem. 272:23528-23531.

7. Ichinohe, T., et al. 1995. Cyclic AMP-insensitive activation of $\mathrm{c}-\mathrm{Src}$ and Syk protein-tyrosine kinases through platelet membrane glycoprotein VI. J. Biol. Chem. 270:28029-28036.

8. Ichinohe, T., et al. 1997. Collagen-stimulated activation of Syk but not c-Src is severely compromised in human platelets lacking membrane glycoprotein VI. J. Biol. Chem. 272:63-68.

9. Poole, A., et al. 1997. The Fc receptor $\gamma$-chain and the tyrosine kinase Syk are essential for activation of mouse platelets by collagen. EMBOJ. 16:2333-2341.

10. Ezumi, Y., Shindoh, K., Tsuji, M., and Takayama, H. 1998. Physical and functional association of the Src family kinases Fyn and Lyn with the collagen receptor glycoprotein VI-Fc receptor $\gamma$-chain complex on human platelets. J. Exp. Med. 188:267-276.

11. Martin, A.M., Kulski, J.K., Witt, C., Pontarotti, P., and Christiansen, F.T. 2002. Leukocyte Ig-like receptor complex (LRC) in mice and men. Trends Immunol. 23:81-88.

12. Sugiyama, T., et al. 1987. A novel platelet aggregating factor found in a patient with defective collagen-induced platelet aggregation and autoimmune thrombocytopenia. Blood. 69:1712-1720.

13. Moroi, M., Jung, S.M., Okuma, M., and Shinmyozu, K. 1989. A patient with platelets deficient in glycoprotein VI that lack both collagen-induced aggre- gation and adhesion. J. Clin. Invest. 84:1440-1445.

14. Nieswandt, B., et al. 2001. Long-term antithrombotic protection by in vivo depletion of platelet glycoprotein VI in mice. J. Exp. Med. 193:459-470.

15. Zheng, Y.M., et al. 2001. Expression of the platelet receptor GPVI confers signaling via the Fc receptor $\gamma$-chain in response to the snake venom convulxin but not to collagen. J. Biol. Chem. 276:12999-13006.

16. Massberg, S., et al. 2003. A crucial role of glycoprotein VI for platelet recruitment to the injured arterial wall in vivo. J. Exp. Med. 197:41-49.

17. Goto, S., et al. 2002. Involvement of glycoprotein VI in platelet thrombus formation on both collagen and von Willebrand factor surfaces under flow conditions. Circulation. 106:266-272.

18. Schulte, V., et al. 2003. Targeting of the collagen-binding site on glycoprotein VI is not essential for in vivo depletion of the receptor. Blood. 101:3948-3952.

19. Boylan, B., et al. 2004. Anti-GPVI-associated ITP: an acquired platelet disorder caused by autoantibody-mediated clearance of the GPVI/FCR $\gamma$-chain complex from the human platelet surface. Blood. 104:1350-1355.

20. Gardiner, E.E., Arthur, J.F., Kahn, M.L., Berndt, M.C., and Andrews, R.K. 2004. Regulation of platelet membrane levels of glycoprotein VI by a plateletderived metalloproteinase. Blood. 104:3611-3617.

21. Stephens, G., et al. 2005. Platelet activation induces metalloproteinase-dependent GP VI cleavage to down-regulate platelet reactivity to collagen. Blood. 105:186-191.

22. Takayama, H., et al. 2005. Characterization of antihuman GPVI autoantibodies mediating persistent target immnunodepletion over a 15 -year period [abstract]. Blood. 106:612a.

23. Suzuki, H., Murasaki, K., Kodama, K., and Takayama, H. 2003. Intracellular localization of glycoprotein VI in human platelets and its surface expression upon activation. Br. J. Haematol. 121:904-912.

24. Kehrel, B., et al. 1998. Glycoprotein VI is a major collagen receptor for platelet activation: it recognizes the platelet-activating quaternary structure of collagen, whereas CD36, glycoprotein IIb/IIIa, and von Willebrand factor do not. Blood. 91:491-499.

25. Ezumi, Y., Kodama, K., Uchiyama, T., and Takayama, H. 2002. Constitutive and functional association of the platelet collagen receptor glycoprotein VI-Fc receptor $\gamma$-chain complex with membrane rafts. Blood. 99:3250-3255.

26. Smethurst, P.A., et al. 2004. Identification of the primary collagen-binding surface on human glycoprotein VI by site-directed mutagenesis and by a blocking phage antibody. Blood. 103:903-911.

27. Lecut, C., et al. 2004. Identification of residues within human glycoprotein VI involved in the binding to collagen: evidence for the existence of distinct binding sites. J. Biol. Chem. 279:52293-52299.

28. Jandrot-Perrus, M., Lagrue, A.H., Okuma, M., and Bon, C. 1997. Adhesion and activation of human platelets induced by convulxin involve glycoprotein VI and integrin $\alpha 2 \beta 1$. J. Biol. Chem. 272:27035-27041.

29. Adie, E.J., et al. 2002. A pH-sensitive fluor, CypHer 5 , used to monitor agonist-induced $\mathrm{G}$ proteincoupled receptor internalization in live cells. Biotechniques. 33:1152-1157.

30. Adie, E.J., et al. 2003. CypHer 5: a generic approach for measuring the activation and trafficking of $\mathrm{G}$ protein-coupled receptors in live cells. Assay Drug Dev. Technol. 1:251-259.

31. Dumont, B., et al. 2006. Chimeric Fc receptors identify ligand binding regions in human glycoprotein VI. J. Mol. Biol. 361:877-887.

32. Horii, K., Kahn, M.L., and Herr, A.B. 2006. Structural basis for platelet collagen responses by the immune-type receptor glycoprotein VI. Blood. 108:936-942.

33. Boylan, B., Berndt, M.C., Kahn, M.L., and Newman, P.J. 2006. Activation-independent, antibody-mediated removal of GPVI from circulating human platelets: development of a novel NOD/SCID mouse model to evaluate the in vivo effectiveness of anti-human platelet agents. Blood. 108:908-914.

34. Rabie, T., et al. 2007. Diverging signaling events control the pathway of GPVI downregulation in vivo. Blood. 110:529-535.

35. Morton, L.F., Hargreaves, P.G., Farndale, R.W., Young, R.D., and Barnes, M.J. 1995. Integrin $\alpha 2 \beta 1$ independent activation of platelets by simple collagen-like peptides: collagen tertiary (triple-helical) and quaternary (polymeric) structures are sufficient alone for a $2 \beta 1$-independent platelet reactivity. Biochem. J. 306:337-344.

36. Mizushima, S., and Nagata, S. 1990. pEF-BOS, a powerful mammalian expression vector. Nucleic Acids Res. 18:5322.

37. Sambrook, J., and Russel, D.W. 2001. Site-specific mutagenesis by overlap extension. In Molecularcloning: a laboratory manual. 3rd edition. Cold Spring Harbor Laboratory Press. Cold Spring Harbor, New York, USA. 13.36-13.39.

38. Nakane, P.K. 1975. Recent progress in the peroxidase-labeled antibody method. Ann. N. Y. Acad. Sci. 254:203-211.

39. Takayama, H., et al. 1991. Ionophore A23187induced protein-tyrosine phosphorylation of human platelets: possible synergism between $\mathrm{Ca} 2+$ mobilization and protein kinase $\mathrm{C}$ activation. Biochem. Biophys. Res. Commun. 174:922-927. 